\title{
Qualidade de Vida no Adulto Queimado: uma revisão sistemática
}

\author{
Fernanda Leite Matos \\ Acadêmica do $4 \stackrel{0}{ }$ ano do curso de Fisioterapia da \\ Escola Bahiana de Medicina e Saúde Pública \\ (fernandamatos01@yahoo.com.br) \\ Ana Luiza Oliveira Barros \\ Fisioterapeuta Especialista em Dermato-Funcional \\ (analuizabarros25@yahoo.com.br) \\ Tipo de Pesquisa \\ Artigo de Revisão Sistemática \\ Origem da Pesquisa \\ Trabalho de Conclusão de Curso de Fisioterapia da \\ Escola Bahiana de Medicina e Saúde Pública \\ Análise do Manuscrito \\ Corpo Editorial da Bahiana \\ Tipo de Análise do Manuscrito \\ Peer Blind Review \\ Recebido em Mai/11 \\ Aprovado em Jun/11
}

\section{Resumo}

Introdução: A queimadura é uma lesão tecidual por resultado da transferência de energia de uma fonte de calor para o organismo humano. No que se refere à QV no sujeito queimado, sabe-se que sua maioria sofre modificações quanto à comunicação dentro do meio social após a lesão. O comprometimento se dá através da dificuldade de lidar com as atividades simples e corriqueiras, que antes concretizavam sem atribuir importância. Objetivo: Realizar uma síntese de pesquisas acerca da QV dos adultos que sofreram queimaduras. Métodos: Trata-se de uma revisão sistemática. Os artigos foram pesquisados na base de dados Medline, SciELO e LILACS. (2005 à abril de 2011), seguindo os critérios: 1) participantes adultos, 2) Participante avaliado após ter sido vítima de lesão térmica, 3) idioma inglês e português, 4) A avaliação da qualidade de vida deveria ser um dos objetivos do artigo, 5) Os artigos deveriam estar disponíveis na íntegra. Resultados: Foram incluídos na pesquisa nove artigos, sendo 7 de perfil quantitativo e 2 qualitativo. Após análise foi constatado que a QV é acometida em diversos aspectos como questões físicas e psicolsociais. Esses estudos se assemelham em alguns prejuízos de domínios em comum como sensibilidade ao calor, e relacionados a trabalho, afeto e imagem corporal. Houve semelhança,também, no que tange a super valorização da vida após o evento da queimadura. Conclusão: 0 estudo evidenciou pela análise do conhecimento publicado que a lesão por forma de queimadura implica negativamente na QV da população afetada e seus familiares.

Palavras-Chave: Qualidade de vida, Queimadura, Queimados 


\title{
Quality of Life in Adult Burned: a systematic review
}

\begin{abstract}
Introduction: A burn is tissue damage resulting from the transfer of energy from a heat source to the human body. With regard to QoL burned in the subject, it is known that most of them with modifications for the communication within the social environment after injury. The commitment is through the difficulty of dealing with the mundane and simple activities, which materialized without giving prior importance. Objective: a synthesis of research on the QOL of adults who have suffered burns. Methods: This is a systematic review. The articles were searched in the Medline, SciELO and LILACS. (2005 to April 2011), the following criteria: 1) adult participants, 2) Participants evaluated after thermal injury victim, 3) English and Portuguese, 4) The assessment of quality of life should be one of the goals of article 5) Articles should be available in its entirety. Results: The study included nine articles, 7 of two qualitative and quantitative profile. After analysis it was found that QOL is affected in several aspects such as physical and psicolsociais issues. These studies are similar in some losses of common areas such as sensitivity to heat, and work-related, affect and body image. There were similar, too, regarding the overrating of life after the burn event. Conclusion: This study showed for the analysis of knowledge that the published form of burn injury implies a negative effect on QoL of the affected population and their families.
\end{abstract}

Keywords: Quality of life, Burns, Burns

\section{Calidad de vida en adultos quemados: una revisión sistemática}

\section{Resumen}

Introducción: Una quemadura es el daño tisular resultante de la transferencia de energía desde una fuente de calor para el cuerpo humano. Con respecto a la calidad de vida se quema en el tema, se sabe que la mayoría de ellos con modificaciones de la comunicación en el entorno social después de la lesión. El compromiso es a través de la dificultad de tratar con las actividades mundanas y simples, que se materializó, sin dar importancia antes. Objetivo: una síntesis de la investigación sobre la calidad de vida de los adultos que han sufrido quemaduras. Métodos: Se realizó una revisión sistemática. Los artículos se realizaron búsquedas en Medline, SciELO, y LILACS. (2005 a abril de 2011), los siguientes criterios: 1) los participantes adultos, 2) Los participantes evaluaron después víctima de lesión térmica, 3) Inglés y Portugués, 4) La evaluación de la calidad de vida debe ser uno de los objetivos de artículos artículo 5) debe estar disponible en su totalidad. Resultados: El estudio incluyó a nueve artículos, siete de los dos el perfil cualitativo y cuantitativo. Tras el análisis se encontró que la calidad de vida se ve afectada en varios aspectos, tales como problemas físicos y psicolsociais. Estos estudios son similares en algunas pérdidas de áreas comunes tales como la sensibilidad al calor, y relacionadas con el trabajo, el afecto y la imagen corporal. No fueron similares, también, con respecto a la sobrevaloración de la vida después de los hechos quema. Conclusión: Este estudio mostró para el análisis del conocimiento que la forma de publicación de la lesión por quemadura implica un efecto negativo en la calidad de vida de la población afectada y sus familias.

Palabras clave: Calidad de vida, Burns, Burns

\section{Qualité de vie pour adultes brûlés: un examen systématique}

\section{Résumé}

Introduction: Une brûlure est dommage tissulaire résultant du transfert d'énergie d'une source de chaleur pour le corps humain. En ce qui concerne la qualité de vie brûlée dans le 
sujet, il est connu que la plupart d'entre eux avec des modifications pour la communication au sein de l'environnement social après une blessure. L'engagement se fait par la difficulté de traiter avec les activités banales et simples, qui se sont matérialisés, sans donner de l'importance préalable. Objectif: une synthèse des recherches sur la qualité de vie des adultes qui ont subi des brûlures. Méthodes: Il s'agit d'un examen systématique. Les articles ont été recherchées dans Medline, SciELO et de lilas. (2005 à avril 2011), les critères suivants: 1) les participants adultes, 2) Les participants ont évalué la victime après une lésion thermique, 3) l'anglais et le portugais, 4) L'évaluation de la qualité de vie devrait être l'un des objectifs de articles article 5) devrait être disponible dans son intégralité. Résultats: L'étude a inclus neuf articles, 7 de deux profil qualitatif et quantitatif. Après analyse, il a été constaté que la qualité de vie est affectée par plusieurs aspects tels que les problèmes physiques et psicolsociais. Ces études sont similaires dans certaines pertes d'espaces communs tels que la sensibilité à la chaleur, et liés au travail, affecter et l'image corporelle. Il y avait même, aussi, concernant la surévaluation de la vie après l'événement brûler. Conclusion: Cette étude a montré pour l'analyse de la connaissance que le formulaire publié de brûlure implique un effet négatif sur la qualité de vie de la population touchée et de leurs familles.

Mots-clés: Qualité de vie, Burns, Burns

\section{Introdução}

A queimadura é uma lesão tecidual por resultado da transferência de energia de uma fonte de calor para o organismo humano, e ocupa o segundo lugar em acidentes que ocorrem mundialmente perdendo apenas para fraturas ${ }^{1,2,3}$. A queimadura quando extensa e profunda representa uma das formas mais graves e complexas de traumatismo 4.

A lesão térmica foi considerada a quarta causa de morte por injúria nos Estados Unidos e a quinta causa de mortes violentas em todo mundo, segundo dados da Organização Mundial de Saúde. No Brasil, estima-se que 1.000 .000 de indivíduos se queimam por ano ${ }^{5}$. A importância da prevenção deste tipo de trauma decorre não só da freqüência com que ocorrem, mas principalmente de sua capacidade de provocar seqüelas funcionais, estéticas e psicológicas $^{6}$. 0 tratamento de pacientes queimados deve ser multidisciplinar e 0 fisioterapeuta é uma peça chave em todas as fases da reabilitação7.

A qualidade de vida (QV) é um termo abrangente que reflete uma condição onde se percebe o grau de satisfação encontrado na vida em geral, incluindo relações amorosas, social e estética existencial. Na saúde, apesar desta relação ser um tanto quanto inespecífica, ela existe. Este conceito tem o valor atribuído à vida, ponderado pelas deteriorações funcionais; percepções e condições sociais que são induzidas pela doença; agravos; tratamentos; bem como a organização política e econômica do sistema assistencial de saúde ${ }^{8}$.

No que se refere à QV no sujeito queimado, sabe-se que sua maioria sofre modificações quanto à comunicação dentro do meio social após a lesão. O comprometimento se dá através da dificuldade de lidar com as atividades de vida diária (AVD's), à volta ao trabalho, relação afetiva e sexual com o conjugue, lazer, limitações físicas e principalmente a perda da autonomia para realizar atividades simples e corriqueiras, que antes concretizavam sem lhes atribuir importância9.

A literatura aponta um conjunto de artigos que tratam da qualidade de vida em pacientes queimados, envolvendo uma avaliação geral da QV após a lesão, o retorno ao trabalho, relação da imagem corporal com limitações psicológicas dentre outros. Contudo, não foi identificado nas bases de dados pesquisadas nenhum estudo que sistematize esse conhecimento nos últimos seis anos. Nesse sentido, o presente trabalho tem o propósito de realizar uma síntese de pesquisas acerca da QV dos adultos que sofreram queimaduras. 


\section{Material \& Método}

Foi realizada uma revisão sistemática acerca da QV em pacientes queimados no período compreendido entre 2005 à abril de 2011. A busca foi realizada em bases de dados eletrônicos de livre acesso e de acesso restrito, quais foram: Medical Literature Analysis and Retrieval System (Medline), Scientific Electronic Library Online (SciELO), Literatura Latino-Americana e do Caribe em Ciências da Saúde (LILACS). Utilizou-se descritores contidos do DECS (Descritores em Ciência da Saúde) e MESH (Medical Subject Headings) nos idiomas: português (qualidade de vida, queimadura, queimados) e inglês (quality of life, burn, burned).

Foram incluídos na pesquisa os artigos que atenderam aos seguintes critérios: 1) participantes adultos, 2) Participante avaliado após ter sido vítima de lesão térmica, 3) idioma inglês e português, 4) A avaliação da qualidade de vida deveria ser um dos objetivos do artigo, 5) Os artigos deveriam estar disponíveis na íntegra.

Foram excluídos os artigos com resumos repetidos ou artigos que foram selecionados pelo título e, porém após leitura do resumo não mantinha relação com o objeto de estudo, isto é, não avaliava a qualidade de vida em pacientes queimados.

A busca pelos artigos evidenciou 532 títulos, porém, logo de início 169 deles, foram excluídos devido ao ano de publicação. Em seguida, dos 363 restantes, 317 foram excluídos pelo título, restando 46 artigos. Nesse grupo os resumos foram lidos, excluindo 19 que não se relacionaram com o objeto de estudo. Dos 27 selecionados para leitura completa, 18 não se adequaram aos critérios de inclusão, restando apenas nove estudos para análise (Figura 1). Os artigos analisados contemplaram sete na língua inglesa e dois na língua portuguesa, os quais foram processados em matrizes contendo as seguintes categorias analíticas: autor, revista, ano, objetivos, metodologia, resultados, instrumento utilizado para avaliação da QV e resumo do texto completo. A análise foi realizada observando a coluna na vertical e posteriormente foram comparados os resultados encontrados entre os estudos.

\section{Resultados}

Aspectos gerais dos artigos encontrados

A Tabela 1 registra as características gerais dos artigos analisados neste estudo. A revisão sistemática acerca da qualidade de vida em adultos queimados apresentou 9 artigos, seguindo os critérios estabelecidos para este estudo. Dos artigos utilizados foram encontrados estudos com propósitos específicos diversos, incluindo a avaliação da QV no paciente queimado.

Dos nove artigos incluídos, quatro $(44,4 \%)$ tinham como objetivo primário apenas a avaliação da QV nessa população ${ }^{9}$ - 12. Os restantes, além da avaliação da QV incluíram, também, outros aspectos, como: retorno ao trabalho13; determinação do peso econômico (custos diretos e indiretos) de vítimas de queimaduras ${ }^{14}$; resultados funcionais em uma população que necessitou de suporte ventilatório15; avaliação a curto e em longo prazo a mortalidade de pacientes com queimaduras graves internada na UTI e com necessidade de suporte ventilatório, além de fatores clínicos no momento da lesão que poderiam prever mortalidade hospitalar16; fatores associados com a probabilidade de retorno ao trabalho após queimaduras e a diferença entre grupos com trabalho e sem em termos de qualidade de vida relacionada à saúde ${ }^{17}$.

No que concerne aos periódicos de publicação, observou-se que, quatro artigos $(44,4 \%)$ concentraram-se em revistas que tratam exclusivamente de pacientes queimados ${ }^{10,13}$-15, os demais trabalhos se inserem em revistas de outras naturezas, tais como: cirurgia plástica ${ }^{12}$, trauma16, reabilitação ${ }^{17}$ e enfermagem ${ }^{9,11}$. 
Quanto à metodologia dos artigos analisados, 77,7\% dos artigos trouxe uma abordagem quantitativa, com aplicação de questionários/escalas de avaliação validados diversos10, 12 16 , e $22,3 \%$ tratavam da questão em abordagem qualitativa. Nesse particular, foram usadas entrevistas elaboradas pelos pesquisadores ${ }^{9,11}$.

Nos estudos com abordagem quantitativa, quatro $(44,4 \%)$ utilizaram a Burn Specific Health Scale-Brief (BSHS-B), sendo que todos eles eram acrescidos de alguma outra forma de avaliação10 13, 15, 17. Dentre elas, observou-se a utilização de parâmetros da queimadura10, questionário de fisioterapia e terapia ocupacional produzido pelos pesquisadores15, coping with burns questionnaire e Pain patient Profile13; e um último abarcou informações como variáveis sociodemográficas, informações pré-queimadura e o The Medical Outcomes Study36-item Short-Form Health Survey (SF-36) ${ }^{17}$. O SF-36 foi utilizado associado a variáveis sociodemográficas e informações sociais em outro estudo12. O European Quality of Life5Dimension (EQ-5D) foi utilizado em 2 estudos o que corresponde 22,2\% dos artigos ${ }^{14,16 .}$

Ressalta-se que nos estudos analisados os questionários são desenhados para avaliar o nível de função relacionado com a QV na saúde. Eles possuem uma determinada quantidade de ítens incluindo domínios bem definidos. Para cada um dos ítens a população estudada deve escolher a melhor resposta, gerando médias que são calculadas para cada um dos domínios. 0 escore final determina alteração na QV. A maior pontuação média indica uma avaliação positiva da função e uma melhor QV.

\section{Qualidade de vida no adulto queimado}

A Tabela 2 mostra os principais achados dos resultados apresentados na literatura sobre a avaliação da QV em adultos queimados. Os resultados dos estudos que avaliam qualidade de vida em vítimas de queimaduras sugerem que existem prejuízos reais diversos. Verifica-se que a lesão parece interferir desfavoravelmente nas dimensões da qualidade de vida, acarretando níveis mais baixos da mesma9-17.

0 estudo de Elsherbiny et al revelou que houve maior comprometimento nos domínios de sensibilidade ao calor, trabalho, afeto e domínios de imagem corporal. Enquanto que os melhores escores foram de relações interpessoais, habilidades simples e função da mão10. Já no estudo de Druery et al os domínios mais comprometidos seguiram uma ordem diferente sendo o mental mais acometido seguido de imagem corporal e afetivo15. 0 primeiro estudo citado também constatou que em sua população, onde a porcentagem de superfície corporal queimada foi maior do que $50 \%$ em todos os casos, quanto maior esse percentual atingido, menor o escore geral da QV10.

Noble et al fez uso de uma população com queimaduras elétricas e revelou que metade das vítimas não sabia do risco na hora da lesão e uma boa parte não usava todo o equipamento de proteção necessário. A maioria dos acidentes aconteceu no ambiente de trabalho, e escores mais baixos foram vistos com relação à satisfação com o mesmo. Participantes com mais de 10\% de superfície corporal queimada (SCQ) apresentaram escores menores nos domínios de função da mão, imagem corporal, sensibilidade ao calor e regimes de tratamento. Neste mesmo estudo, também foi pesquisado, a resposta após cinco anos de lesão onde houve melhora considerável nos domínios físicos, mas continuaram mostrando diminuição nos escores de afeto, sexualidade e relações interpessoais ${ }^{13}$.

Sanchez et al utilizou o EQ-5D para medir a QV média, onde na verdade foram classificados de acordo com os diagnósticos relacionados com graus e constatou que a qualidade de vida diminuiu a medida que a gravidade da queimadura aumentou ${ }^{14}$.

Pavoni et al constatou que os indivíduos possuíam uma saúde melhor antes da lesão e os sintomas mais freqüentes apresentados após a queimadura eram ansiedade e depressão, seguidos de auto-cuidado e dor e problemas de mobilidade. Os problemas com a realização de AVD's eram constantes, e nenhum dos participantes retornou ao trabalho' ${ }^{16 .}$

0 estudo de Dyster-Aas et al analisou separadamente os grupos que estavam trabalhando e os que não tinha estavam. O grupo do trabalho pontuou mais nos domínios e 
subdomínios de funcionamento físico (função física) e corporais (dor) e tiveram médias mais elevadas nas subescalas seguintes, no domínio mental, função social e saúde mental. Quanto à imagem corporal os grupos não tiveram diferença ${ }^{17}$.

A investigação realizada por Souza et al dividiu a população de estudo em dois grupos, por tempo de internamento (mais de 14 dias ou 14 dias ou menos), e constatou que quanto menor o tempo de internação, maior a QV. Contudo, o ambiente hospitalar piorou percepção do indivíduo em relação à sua QV, nos domínios aspecto físico, aspecto emocional e estado geral de saúde.

Pode-se, portanto, observar que há uma relação entre o tempo de internamento e a QV relatada pelos participantes. Com relação ao sexo, os homens apresentaram médias maiores nos domínios capacidade funcional e dor, enquanto as mulheres apresentaram maior média no domínio aspecto social12.

Quanto aos artigos de perfil qualitativo, o trabalho de Moi et al concluiu que o estresse, trabalho, fadiga ou falta de atenção, contribuíram para o acontecimento das queimaduras. Sendo que a recomposição do ambiente e do seu corpo eram os primeiros motivos de inquietação. A seriedade do evento foi evidenciada rapidamente para todos os entrevistados, em especial quando perceberam as seqüelas físicas. A dor aguda não foi indicador de gravidade da queimadura, e inclusive alguns dos inquiridos tentaram manter-se no controle da situação até a chegada dos primeiros socorros. Depois do acidente, a memória ficou comprometida, isto pôde ser percebido porque eles já não conseguiam traçar uma ordem cronológica dos fatos ${ }^{11}$.

Este trabalho também evidenciou a dificuldade de lidar com as seqüelas da queimadura, ressaltando que a capacidade de aceitação da lesão foi essencial para um melhor prognóstico. Interpretar o sofrimento como necessário para haver melhora ajudou na nesta fase. 0 acidente fez com que o paciente pudesse encarar a morte como algo próximo. Desta forma, a sobrevivência e progresso eram tratados como benção e com todas as limitações apresentadas, os pacientes apenas sentiam-se agraciados pela sobrevivência11.

Costa et al buscando interpretar os significados de QV de um grupo de pessoas em processo de reabilitação de queimaduras, constatou que o trauma está associado diretamente à dor, insegurança, risco de morte, e ao ambiente hospitalar. Além disso, houve também preocupações com as posteriores deformidades e limitações físicas, possíveis empecilhos para realização de atividades em geral. As reações ao trauma foram variáveis, desde sobrecarregar de sentido afirmativo, até negar à experiência da queimadura. Houve relatos de que após a queimadura foi percebido uma maior reflexão a respeito da vida, sendo este marco para transformação de valores?.

o mesmo autor buscou saber dos pacientes os sentidos atribuídos a "vida boa" em diferentes aspectos. Quanto à felicidade os entrevistados relacionam: ter saúde, emprego, conforto, bom relacionamento familiar e maior tempo para descansar. Associados com satisfação destacam-se: ausência de defeitos físicos, amigos, bom relacionamento familiar, saúde, capacidade de trabalhar, conforto material. Quanto ao sucesso pessoal: o trabalho, força de vontade, crescer na vida, satisfação de necessidades materiais e autonomia foram citados. Por fim, a realização pessoal foi atrelada a independência, ter emprego, saúde, ausência de cicatrizes, casa própria, satisfazer as necessidades familiares e conformar-se com as limitações decorrentes da queimadura9.

0 trabalho também apontou que a maioria dos sujeitos demonstra descontentamento com a aparência física alterada pelas seqüelas da queimadura, repercutindo na auto-imagem. Isto é, dificuldades relativas ao trabalho, à locomoção e aos relacionamentos, prioritariamente familiar e sexual. Para a maioria dos participantes da pesquisa, a queimadura é um marco que separa uma vida sadia de outra sem saúde, nos homens, a perda da saúde ocasionada pela queimadura, destaca-se o comprometimento do desempenho sexual. Constatou-se também que após a queimadura, os sujeitos, com raras exceções, tornaram-se menos comunicativos 
dentro de seu grupo social ou, simplesmente, se afastaram do convívio social fora do espaço doméstico?.

\section{Discussão}

0 presente trabalho pretendeu obter a melhor evidência quanto à QV em pacientes adultos, vítimas de queimaduras. Para tanto, analisou nove estudos a esse respeito. Embora o número de títulos oriundos das buscas bibliográficas não seja pequeno (512 artigos), ele é reduzido quando feita a seleção para o objetivo proposto

A análise dos resultados dos artigos selecionados demonstrou algumas semelhanças. Quanto ao agente causal das queimaduras a maioria foi de origem térmica, seguida de queimaduras elétricas $10,11,14,15$. Importante salientar que dois desses artigos chamaram atenção para ocorrência de uma grande parte desses eventos em domicílio, e de forma acidental10, 11. Isto pode ser ratificado com estudos sobre perfil epidemiológico de adultos com queimaduras os quais confirmam os achados do presente estudo $0^{5,18}$.

Após a queimadura, diversos aspectos são alterados na vida das vítimas. Imagem corporal, habilidades físicas funcionais e questões relacionadas às relações interpessoais foram abordadas em diversos artigos como comprometidas gravemente9, 10, 13, 15, 17. Queimaduras graves são consideradas danosas à saúde psicológica e global10, podendo gerar uma crise psicológica e prejuízos à auto-estima ${ }^{19}$. A construção da imagem corporal quando se tratando de uma pessoa com deficiência ou limitação se deve a percepção de um corpo desvantajoso em relação aos outros ditos normais. As transformações na imagem corporal levam a conflitos que necessitam de aceitação das diferenças em relação ao padrão social de corpo perfeito. Além disso, é comum que limitações físicas causem certa fragilidade emocional e de relacionamento com outras pessoas, em especial quando essas pessoas não têm as mesmas limitações ${ }^{20}$.

Em longo prazo, as limitações físicas aparecem não mais como um problema principal, um dos artigos relata melhora significativa neste aspecto. Isto pode ser explicado pela adaptação dos pacientes para realização de suas atividades dentro de suas limitações ${ }^{13}$. Entretanto, existem outros fatores como a dor, que apareceu em outro artigo como presente na maioria dos acometidos em longo prazo. Este parece influenciar diretamente na saúde física e psicológica, podendo dificultar a realização de atividades cotidianas, e, portanto, é importante gerador de incapacidade ${ }^{16}$.

A dor apareceu novamente nos trabalhos de perfil qualitativo e de forma contraditória. Em um dos trabalhos ela foi relacionada diretamente ao trauma chegando a ser referida como insuportável9. Em outro, chegou a ser relatada como ausente nos momentos iniciais, não sendo, portanto indicativo de gravidade ${ }^{11}$. A dor é uma sensação inespecífica e o paciente pode reagir de diversas formas sejam elas: ignorando-a, fazendo atribuição da realidade ou super valorizando-a ${ }^{21}$. Desta forma, pode-se prever que não é certo subestimar a dor do paciente em função da reação apresentada, mas sim procurar entender e avaliar como agir nessa situação.

Estudos se assemelharam quanto à relação entre o tamanho e gravidade da queimadura e a sua implicabilidade na QV10,13,15-17. Considerando que as lesões térmicas são altamente lesivas e incapacitantes, quanto maior a alteração estrutural na cobertura cutânea, maior será a implicabilidade negativa na vida do indivíduo e maior o comprometimento da QV. Além disso, a extensão e grau da queimadura também estão associados ao tempo de internamento hospitalar, que apareceu em um dos estudos como sendo preditor para uma pior apresentação nos escores de QV12. Neste próprio artigo o autor cita que a hospitalização é um agente que pode gerar efeitos diversos e depende de uma série de fatores como a idade do indivíduo, intensidade das queimaduras, da eficiência dos profissionais envolvidos e também da maneira como a família e o próprio indivíduo a gerencia. Visto isso, os resultados obtidos 
nos estudos sofreram influência de acordo com o tempo de queimadura, a extensão e o grau da lesão, uma vez que, um dos estudos constatou através de seus resultados que a QV diminuiu à medida que a gravidade da queimadura aumentou ${ }^{14}$.

A sexualidade foi outro aspecto apontado como acometido negativamente após a lesão" 13,17 , onde os homens apareceram com maior descontentamento9. Autores relataram que a sexualidade é um contexto amplo onde se faz importante o desejo, os indivíduos queimados com todo prejuízo gerado em seu físico e imagem corporal, tem este fator comprometido. Os homens por terem características gerais relacionadas à atitude e desempenho se sentem mais frustrados e por isso tem descontentamento maior ${ }^{20}$.

Fatores relacionados ao labor apareceram de forma convergente em diversas investigações abordadas neste estudo. 0 retorno à ocupação foi associado de forma positiva a QV17, no entanto, as literaturas analisadas demonstraram que a maioria dos pacientes não conseguia retornar ao trabalho após a lesão, ou este tempo de retorno era demasiadamente grande $\mathrm{e}^{9}, 10,13,17$. Artigos existentes abordando este aspecto revelam que a queimadura gera um nível tamanho de comprometimento que implica na impossibilidade de retorno ao trabalho ou prazo extenso no tempo de recuperação13,14,22. Muito embora, este último tenha sido associado também ao fato de a população estar sendo beneficiada por seguro médico ${ }^{14}$.

Outra questão interessante foi à sensibilidade ao calor. Esta se mostrou comum após este tipo de lesão sendo ponto importante, avaliado nos questionários específicos de QV em queimados ${ }^{9,13}$.

Houve semelhança encontrada no que tange a super valorização da vida após o evento da queimadura9, 11. A literatura concorda com esses dados evidenciando que o paciente adquire com o passar do tempo sentimentos de autoconfiança no processo de internação prolongada e isto, pré dispõe a uma maior reflexão sobre a própria existência ${ }^{9,23}$.

Este estudo mostrou pela análise do conhecimento publicado que a lesão por forma de queimadura implica negativamente na QV da população afetada e seus familiares. Observa-se a necessidade de programas de prevenção contra lesões térmicas, uma vez que a maior parte da população acometida sofreu o acidente em casa e de forma acidental, ou no trabalho por falta de proteção.

\section{Conclusão}

Esta investigação mostrou, que a concentração da produção científica no âmbito qualitativo é baixa. Isto impossibilita a avaliação da QV em aspectos outros, a exemplo dos sentimentos das vítimas mediante aos problemas e sua influência na vida após o trauma. Nesse sentido, sugere-se novas pesquisas que abordem o tema especialmente na perspectiva qualitativa. No entanto, caso haja esforcos na abordagem quantitativa parece interessante 0 uso de grupo controle para uma melhor comparação sobre o acometimento da QV na população queimada.

\section{Referências}

1. SILVA, R M A; \& CASTILHOS, A P L. "A identificação de diagnósticos de enfermagem em paciente considerado grande queimado: um facilitador para implementação das ações de enfermagem", Rio de Janeiro-RJ: Revista Brasileira de Queimaduras, 2(9): 60-65. 2010.

2. COSTA, A S; FREITAS, A M; \& SANTOS, C O. “Efeitos da aplicação de microcorrente no processo de reparo tecidual de queimaduras. Salvador-BA: Revista Fisioterapia Brasil, 11(2): 103-08. 2010.

3. PEREIRA, R M; “Efeitos de lasers de baixa potencia em três diferentes comprimentos de onda no processo de cicatrização de queimadura de 3 grau", São Jose dos Campos-SP: 2005. (Disponível em $<$ www.dominiopublico.gov.br/pesquisa/DetalheObraForm.do?select_action=\&co_obra=122459. Acesso em 30/01/2011). 
4. O'SULLIVAN, S B; \& SCHMITZ, T J. Queimaduras. Fisioterapia Avaliação e Tratamento. (4th ed), Barueri- SP: Manole, pp 845-871.

5. SILVA, G P F; et al. “Estudo epidemiológico dos pacientes idosos queimados no centro de tratamento de queimados do hospital instituto doutor José frota do município de Fortaleza-CE, no período de 2004 a 2008", Fortaleza-CE: Revista Brasileira de Queimaduras, 9(1): 7-10. 2010.

6. COUTINHO, B B A; et al. "Perfil epidemiológico de pacientes internados na enfermaria de queimados da associação beneficente de Campo Grande Santa Casa-MS", Campo Grande-MS: Revista Brasileira de Queimaduras, 9(2): 50-53. 2010.

7. MILANI, G B; JOÃO, S M A; \& FARAH, E A. "Fundamentos da Fisioterapia dermato-funcional: revisão de literatura", São Paulo-SP: Fisioterapia e Pesquisa, 3(12): 37-46. 2006.

8. MINAYO, M C S; HARTZ, Z M A; \& BUSS, P M. "Qualidade de vida e saúde: um debate necessário", Rio de Janeiro-RJ: Ciência \& Saúde Coletiva, 5(1): 7-18. 2000.

9. COSTA, M.C.S. et al. Significados De Qualidade De Vida: Análise Interpretativa Baseada Na Experiência De Pessoas Em Reabilitação De Queimaduras. Revista Latino-americana de Enfermagem, Ribeirão Preto - SP, v.16, n.2, 2008.

10.ELSHERBINY, O E E. et al. "Quality of life of adult patients with severe burns", Burns, 37(5): 775-88. 2011.

11. MOI, A L; VINDENES, H A; \& JENGEDAL, E. "The experience of life after burn injury: a new bodily awareness", Bergen: Journal of Advanced Nursing, 64(3): 278-86. 2006.

12.SOUZA, T J A. "Qualidade de vida do paciente internado em uma unidade de queimados", Campo Grande-MS: Revista Brasileira de Cirurgia Plástica, 26(1): 10-15. 2011.

13. NOBLE, J; GOMES, M; FISH, J S. "Quality of life and return to work following electrical burns", Burns, 32(2): $159-64.2006$.

14.SANCHEZ, J L A. et al. "Socio-economic cost and health-related quality of life of burn victims in Spain", Burns, 34(7): 975-81. 2008.

15.DRUERY, M; BROWN, T L H B; \& MULLER, M. “Long term functional outcomes and quality of life following severe burn injury", Burns, 31(6): 692-95. 2005.

16.PAVONI, V. et al. "Outcome predictors and quality of life of severe burn patients admitted to intensive care unit", Scandinavian Journal of Trauma, Resuscitation and emergency Medicine, 18(1): 18-24. 2010.

17. DYSTER-AAS, J; KILDAL, M; \& WILLEBRAND, M. "Return to work and health-related quality of life after burn injury", Journal of Rehabilitation Medicine, 39(1): 49-55. 2007.

18. COUTINHO, B B A. "Perfil epidemiológico de pacientes internados na enfermaria de queimados da Associação Beneficente de Campo Grande Santa Casa-MS", Revista Brasileira de Cirurgia Plástica, 9(2): 50-53. 2010.

19. KNIGHTON, J. Nursing management: burns. Medical surgical nursing: assessment and management of clinical problems. (7th ed), Philadelphia: Mosby \& Elsevier, 2007. pp. 483-507.

20. MAIA, A C B. "A importância das relações familiares para a sexualidade e a autoestima de pessoas com deficiência física", 2010. (Disponível em <www.psicologia.com.pt> Acesso em 03/07/2011).

21.ARTZ, C P; MONCRIEF, J A; \& PRUIT, B A. Queimaduras. Rio de Janeiro-RJ: Interamericana, 1980.

22. BRYCH, S B. et al. "Time off work and return to work rates after burns: systematic review of the literature and a large two-center series", Journal of Burn Care \& Rehabilitation, 22(6): 401-05. 2001.

23.SOLTANI, K; ZAND, R; \& MIRGHASEMI, A. "Epidemiology and mortality of burns in Tehran", Iran: Burns. 24(4): 325-28. 1998.

\section{Apêndice}




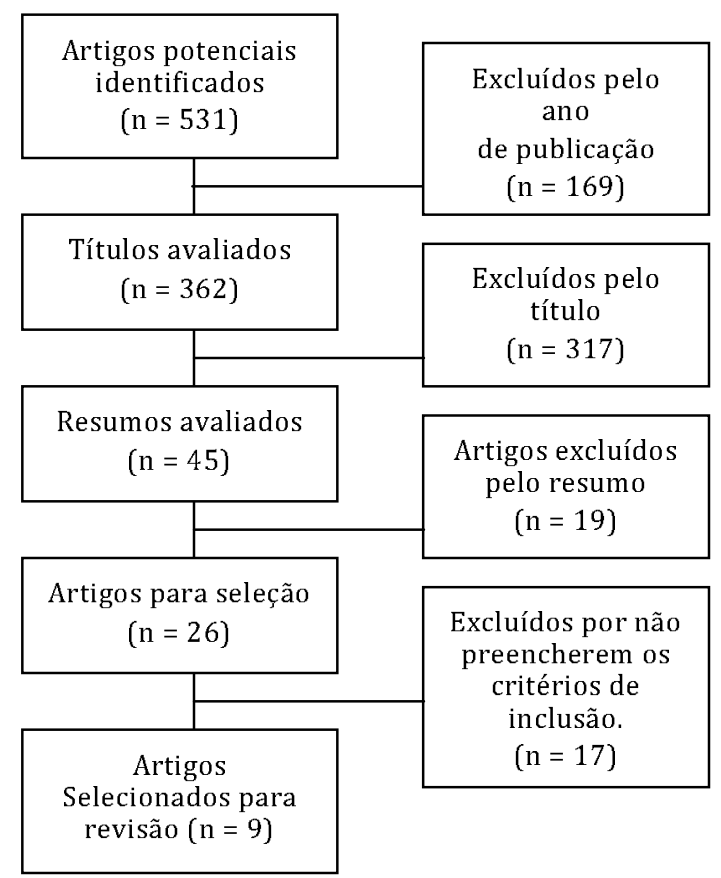

Figura 1 - Fluxograma com descrição do procedimento para seleção dos artigos acerca do tema QV no adulto queimado

Tabela 1

Referente aos Aspectos Gerais dos Artigos que Avaliam a Qualidade de Vida no Adulto Queimado nos Entre 2005 e Abr-2011

\begin{tabular}{|c|c|c|c|c|}
\hline Artigos/ Ano & $\begin{array}{l}\text { Titulo do } \\
\text { Periódico }\end{array}$ & Objetivo & Metodologia & $\begin{array}{l}\text { Instrumento } \\
\text { de Avaliação }\end{array}$ \\
\hline $\begin{array}{l}\text { Costa, } \\
\text { et al. / } 2008^{9}\end{array}$ & $\begin{array}{l}\text { Revista Latino } \\
\text { americana de } \\
\text { Enfermagem }\end{array}$ & $\begin{array}{c}\text { Contribuir para a } \\
\text { interpretação dos } \\
\text { significados de QV de um } \\
\text { grupo de pessoas em } \\
\text { processo de reabilitação de } \\
\text { queimaduras, a partir de } \\
\text { suas experiências, } \\
\text { concepções e valores. }\end{array}$ & Qualitativo & Entrevista \\
\hline $\begin{array}{l}\text { Elsherbiny, } \\
\text { et al. / } 201110\end{array}$ & Burns & $\begin{array}{c}\text { Avaliar a QV de adultos com } \\
\text { queimaduras graves. }\end{array}$ & Quantitativo & $\begin{array}{l}\text { Parâmetros de } \\
\text { queimadura } \\
\text { BSHS-B }\end{array}$ \\
\hline $\begin{array}{l}\text { Moi et al. / } \\
200811\end{array}$ & $\begin{array}{l}\text { Journal of } \\
\text { Advanced } \\
\text { Nursing }\end{array}$ & $\begin{array}{c}\text { Explorar e descrever a } \\
\text { experiência da QV após uma } \\
\text { grande queimadura usando } \\
\text { uma abordagem } \\
\text { fenomenológica. }\end{array}$ & Qualitativo & $\begin{array}{c}\text { Variáveis sócio- } \\
\text { demográficas } \\
\text { Entrevista }\end{array}$ \\
\hline $\begin{array}{l}\text { Souza, } \\
\text { et al. / } 201112\end{array}$ & $\begin{array}{l}\text { Revista } \\
\text { Brasileira de } \\
\text { Cirurgia } \\
\text { Plástica }\end{array}$ & $\begin{array}{c}\text { Verificar quais domínios de } \\
\text { QV desses pacientes } \\
\text { estavam mais } \\
\text { comprometidos ou }\end{array}$ & Quantitativo & $\begin{array}{c}\text { Caracterização sócio } \\
\text { demográfica } \\
\text { SF-36 }\end{array}$ \\
\hline
\end{tabular}




\begin{tabular}{|c|c|c|c|c|}
\hline & & $\begin{array}{l}\text { preservados, durante a } \\
\text { hospitalização. }\end{array}$ & & \\
\hline $\begin{array}{l}\text { Noble, } \\
\text { et al. / } 2006^{13}\end{array}$ & Burns & $\begin{array}{c}\text { Investigar a QV e retorno ao } \\
\text { trabalho dos pacientes que } \\
\text { sofrem queimaduras } \\
\text { elétricas. }\end{array}$ & Quantitativo & $\begin{array}{c}\text { BSHB-B } \\
\text { Coping with Burns } \\
\text { questionnaire } \\
\text { Pain Patient Profile. }\end{array}$ \\
\hline $\begin{array}{l}\text { Sanchez, } \\
\text { et al. / } 2007^{14}\end{array}$ & Burns & $\begin{array}{c}\text { Determinar o peso } \\
\text { econômico (custos diretos e } \\
\text { indiretos) de vítimas de } \\
\text { queimaduras e o impacto da } \\
\text { queimadura na saúde e } \mathrm{QV} \\
\text { na Espanha. }\end{array}$ & Quantitativo & EQ-5D \\
\hline $\begin{array}{l}\text { Druery, } \\
\text { et al. / } 2005^{15}\end{array}$ & Burns & $\begin{array}{c}\text { Em longo prazo, avaliar a } \\
\text { QV e resultados } \\
\text { funcionais entre os } \\
\text { indivíduos que sobreviveu } \\
\text { a uma grave e necessitaram } \\
\text { de suporte ventilatório. }\end{array}$ & Quantitativo & $\begin{array}{c}\text { BSHS- B } \\
\text { Questionário } \\
\text { complementar de } \\
\text { fisioterapia e Terapia } \\
\text { Ocupacional }\end{array}$ \\
\hline $\begin{array}{l}\text { Pavoni, } \\
\text { et al. / } 2010^{16}\end{array}$ & $\begin{array}{l}\text { Scandinavian } \\
\text { Journal of } \\
\text { Trauma, } \\
\text { Resuscitation } \\
\text { and } \\
\text { Emergency } \\
\text { Medicine }\end{array}$ & $\begin{array}{c}\text { Avaliar a curto longo prazo } \\
\text { a mortalidade de } \\
\text { pacientes com queimaduras } \\
\text { graves, internada na UTI } \\
\text { com necessidade de suporte } \\
\text { ventilatório. } \\
\text { Identificar fatores clínicos } \\
\text { que poderiam prever } \\
\text { mortalidade hospitalar. } \\
\text { Determinar a QV } \\
\text { relacionada à saúde um ano } \\
\text { depois da lesão, utilizando o } \\
\text { questionário EQ-5D. }\end{array}$ & Quantitativo & EQ-5D \\
\hline $\begin{array}{c}\text { Dyster-Aas et al. / } \\
200717\end{array}$ & $\begin{array}{c}\text { Journal of } \\
\text { Rehabilitation } \\
\text { Medicine }\end{array}$ & $\begin{array}{l}\text { Avaliar quais fatores estão } \\
\text { associados com a } \\
\text { probabilidade de retorno ao } \\
\text { trabalho. } \\
\text { Investigar o que diferencia o } \\
\text { grupo que retornou ao } \\
\text { trabalho do grupo que não } \\
\text { tem em termos de QV } \\
\text { Relacionada à Saúde. }\end{array}$ & Quantitativo & $\begin{array}{c}\text { Informações pré- } \\
\text { queimadura } \\
\text { Variáveis sociodemo- } \\
\text { gráficas } \\
\text { Escalas de } \\
\text { Personalidade (SSP). } \\
\text { SF-36 } \\
\text { BSHS-B) }\end{array}$ \\
\hline
\end{tabular}

OBS: Compilação das autoras.

Tabela 2

Referente aos Principais Achado dos Resultados da Avaliação da Qualidade de Vida no Adulto Queimado de Artigos Selecionados Entre 2005 e Abr-2011

\begin{tabular}{|l|l|}
\hline \multicolumn{1}{|c|}{ Artigos } & \multicolumn{1}{c|}{ Principais Achados } \\
\hline \multirow{4}{*}{$\begin{array}{l}\text { Costa, } \\
\text { et } a \text { l. }\end{array}$} & $\begin{array}{l}\text { O trauma foi associado à dor, insegurança, risco de morte, ao ambiente hospitalar e as } \\
\text { limitações físicas; } \\
\text { Após a queimadura foi percebido uma maior reflexão a respeito da vida, sendo este } \\
\text { marco para transformação de valores. } \\
\text { A maioria dos entrevistados demonstra descontentamento com a aparência física que } \\
\text { acaba por repercutir nas relações sociais, sexuais e profissionais do mesmo. } \\
\text { A queimadura é associada vida sem saúde. }\end{array}$ \\
\hline Elsherbiny, & Menor pontuação: sensibilidade ao calor, trabalho, afeto e domínios de imagem \\
\hline
\end{tabular}




\begin{tabular}{|c|c|}
\hline et al. ${ }^{10}$ & $\begin{array}{l}\text { Corporal. } \\
\text { Melhores escores foram de relações interpessoais, habilidades simples e função da } \\
\text { mão. } \\
\text { Quanto maior a SCQ da queimadura menor o escore de QV. }\end{array}$ \\
\hline $\begin{array}{l}\text { Moi, } \\
\text { et al. } 11\end{array}$ & $\begin{array}{l}\text { Estresse, trabalho, fadiga ou falta de atenção, contribuíram para o acidente; } \\
\text { As vítimas tiveram dificuldade de lidar com as físicas, onde a aceitação do problema e } \\
\text { do sofrimento como um "mal necessário" foi indicativo de um melhor prognóstico; } \\
\text { A queimadura contribuiu para que as vítimas tivessem um olhar diferenciado para com } \\
\text { a vida, agradecendo pela sobrevivência; }\end{array}$ \\
\hline $\begin{array}{l}\text { Souza, } \\
\text { et al. } 12\end{array}$ & $\begin{array}{l}\text { Constata que quanto menor o tempo de internação, maior a } Q V \text {. } \\
\text { O ambiente hospitalar é maléfico para percepção do indivíduo em relação à sua } Q V \text {, nos } \\
\text { domínios aspecto físico, aspecto emocional e estado geral de saúde. }\end{array}$ \\
\hline $\begin{array}{l}\text { Noble, } \\
\text { et al. }{ }^{13}\end{array}$ & $\begin{array}{l}\text { Menor pontuação: relacionados à satisfação com o trabalho. } \\
\text { Análise a longo prazo: Constatou que houve melhora nos domínios físicos, porém } \\
\text { persiste a diminuição nos escores de afeto, sexualidade e relações interpessoais. }\end{array}$ \\
\hline $\begin{array}{l}\text { Sanchez, } \\
\text { et al. }{ }^{14}\end{array}$ & $\begin{array}{l}\text { Constata que a qualidade de vida diminuiu à medida que a gravidade da queimadura } \\
\text { aumentou. }\end{array}$ \\
\hline $\begin{array}{l}\text { Druery, } \\
\text { et al. } 15\end{array}$ & Menor pontuação: domínio mental, imagem corporal e afetivo. \\
\hline $\begin{array}{l}\text { Pavoni, } \\
\text { et al. } 16\end{array}$ & $\begin{array}{l}\text { Constatou que a lesão térmica influenciou negativamente na saúde dos acometidos. } \\
\text { Sintomas frequentes: ansiedade e depressão; auto-cuidado e dor e problemas de } \\
\text { mobilidade. } \\
100 \% \text { dos participantes não retornou ao trabalho. }\end{array}$ \\
\hline $\begin{array}{l}\text { Dyster- } \Lambda \text { as, } \\
\text { et al. } 17\end{array}$ & $\begin{array}{l}\text { Grupo do trabalho gerou maiores escores nos domínios e subdomínios de } \\
\text { funcionamento físico e corporais e tiveram médias mais elevadas nas subescalas no } \\
\text { domínio mental, função social e saúde mental. } \\
\text { Os grupos não diferiram quanto à imagem corporal. }\end{array}$ \\
\hline
\end{tabular}

OBS: Compilação das autoras. 